\title{
The argument from correlation to cause in science communication
}

\author{
Martijn H. DEMOLLIN*
}

\begin{abstract}
A main activity within popularized science communication is the expert-to-layman transferral of scientific knowledge. Correlative connections have proven to be a problematic concept to adequately communicate and form a relatively common source for the misrepresentation of scientific knowledge. Depending on the strength of the causal claim, such an inferential step can be considered an "argument from correlation to cause". This paper reconstructs the argumentative pattern that is typical for these arguments and proposes a number of critical questions for their evaluation. Finally, an analysis is presented of a natural example of reasoning from correlational evidence to cause within the context of popularized science.
\end{abstract}

\section{KEYWORDS}

argumentative pattern; cause; correlation; critical questions; popularized science; pragma-dialectics; reasonableness; science communication

* Ph.D. researcher at the Faculty of Administration and Social Science in the Warsaw University of Technology, Poland, and a member of the Laboratory of The New Ethos at the International Centre for Formal Ontology (ICFO). E-mail: martijn.demollin@pw.edu.pl. 


\section{INTRODUCTION}

The popularization of scientific research - also called accommodated science writing - is defined as the process of making scientific findings and publications accessible to a more general public. Popular scientific discourse is often characterized by a less technical vernacular than scientific literature, as a professional medium, and it generally aims to both inform and persuade non-scientific outsiders. Popularizing scientific knowledge has been traditionally considered an activity situated outside of the academic domain. It was generally deemed unrelated to the process of knowledge production, or the validation of scientific findings, both within academic circles and beyond (Shinn \& Whitley, 1985: 3). However, in more recent developments, science popularization has become progressively widespread and connected to the process of validating scientific knowledge towards society at large. In part, this movement is due to a greater public and political emphasis on the amenability of scientific research, which stresses the societal importance and potential application of scientific findings outside of the academic sphere (Jamieson, Kahan, \& Scheufele, 2017).

Expert-to-expert scientific literature is generally characterized by the presence of tentative, careful and qualified propositions of fact or relation. Popular science tends to present scientific research and its corresponding claims with less reluctance. Expert-to-expert scientific publications, within the academic domain, are aimed at facilitating a number of institutional goals: reporting informatively and persuasively on scientific progress; the accumulation of scientific knowledge; and the critical examination of scientific claims by peers. In contrast, popularized scientific texts fulfil a role in the academic domain and the journalistic domain. As such, popular scientific discourse can be considered as its own unique communicative activity type (van Eemeren, 2010) with its own set of characteristics and discursive attributes. This makes the translative process from scientific discourse into a popular scientific publication interesting from an argumentation-theoretical point of view. Expert-to-expert and popularized scientific publications serve to fulfil dissimilar institutional goals and cater to different audiences, which affects the argumentative strategies employed in each respective genre.

The argument from correlation to cause is a good example of a type of reasoning that is often employed with considerable restraint in professional scientific discourse, but less so in popularized scientific texts. While correlations possess certain predictive capacities, they may only be employed to infer causation under very strict conditions, which is due to the fact that there could remain a number of hidden influences that may play a part in the observed relation between two variables. In some cases, one could argue that a particular correlation does point to a causal link between the observed variables, 
when the presence of one variable is believed to directly influence the presence of another. When a correlative argument is adduced in defence of a claim to the existence of a causal relation, this is also known as an argument from correlation to cause (Walton, 1996: 71). In order for such an argument to be acceptable, several other arguments, aside from the correlative connection between the observed variables, are needed. Employing a correlation in an argument supporting a causal relation can be perfectly reasonable from an argumentative point of view - if there is sufficient additional evidence to support the causal claim. However, this type of argumentation has proven to be problematic within the context of popular science communication and can lead to the misrepresentation of scientific knowledge to the greater public. To allow for the critical assessment of such arguments in natural language, it is fruitful to determine how they can be best evaluated, and which critical questions are most essential for their judgement.

In order to further explore these arguments from correlation to cause in the context of popular science, this paper will: (a) provide a characterization of popular scientific discourse as a communicative activity type, (b) formulate the expected argumentative pattern for arguments from correlation to cause and the critical questions that can be posed to test this type of argument, and (c) discuss a case study of such argumentation within the context of popularized science communication.

\section{CHARACTERIZING POPULAR SCIENTIFIC DISCOURSE AS A COMMUNICATIVE ACTIVITY TYPE}

Most popular scientific texts are situated between the realms of academia and journalism, which means they aim to fulfil institutional goals relevant to both these communicative domains. Popularized scientific discourse that is intended for a general public is also called expert-to-layman communication and constitutes a unique communicative activity type. As Frans van Eemeren notes, when attempting to distinguish communicative activity types from one another, it is important to describe "the specific goals they are to serve in order to fulfil their mission in realizing the institutional point of the communicative activity" (van Eemeren, 2010: 144). Popular scientific discourse can be categorized among the communicative activity types that "prototypically involve the activation of more genres of conventionalized communicative practices" (van Eemeren, 2010: 144). This means popular scientific discourse functions as a hybrid communicative activity type. Table 1 below presents an overview of my formulation of the institutional goals popular scientific texts aim to achieve within the journalistic and academic domains. 
The institutional goals of popular scientific discourse have some overlap to those of professional scientific publications. One of the primary goals of popular scientific texts is informing the general public on scientific findings by providing a representative interpretation of the scientific source material and providing some argumentation for how this scientific knowledge came into being (Goal $1 \& 2$, Table 1). However, the goal of critical examination and evaluation of scientific findings carries less focus within popular scientific discourse, given the fact that it generally does not target a purely academic audience, but a considerably broader audience. Additionally, it is likely that scientific knowledge that is popularized will have already been subjected to the process of critical review by scientific peers elsewhere, before being disseminated to a wider public.

Table 1: Institutional goals of the communicative activity type "popular scientific discourse"

\begin{tabular}{cll}
\hline $\begin{array}{c}\text { Communicative } \\
\text { Activity Type }\end{array}$ & Communicative Domain & \multicolumn{1}{c}{ Institutional Goals } \\
\hline & Scholarly communication & $\begin{array}{l}\text { 1. Providing an adequate and accurate } \\
\text { representation of scientific findings. }\end{array}$ \\
\cline { 3 - 3 } $\begin{array}{c}\text { Popular scientific } \begin{array}{l}\text { 2. Providing argumentation for the } \\
\text { discourse } \\
$\cline { 2 - 3 }\end{array} \\
\text {$knowled interpretation of scientific }\end{array}$ \\
\hline \multirow{2}{*}{ Journalistic communication } \\
\cline { 2 - 2 } & $\begin{array}{l}\text { 3. Simplifying the scientific source } \\
\text { material in order to make it accessible } \\
\text { to a wider audience. }\end{array}$ \\
$\begin{array}{l}\text { 4. Indicating the societal importance } \\
\text { and significance of the scientific } \\
\text { knowledge reported on. }\end{array}$ \\
\hline
\end{tabular}

Another important factor influencing the goals of popular scientific discourse is that its content is bound to professional scientific material already in existence. Authors of popular scientific texts have an institutional responsibility to provide a representation of existing scientific knowledge that is both adequate and reasonable, although it does not have to be complete or exhaustive. If a popular scientific text strays too far from the source material, its author runs the risk of misrepresenting the scientific material it is based on, possibly leading to the popularized scientific article being rescinded. However, there is no absolute delineation of what constitutes a reasonable or unreasonable representation of scientific source material. Also, authors of 
popular scientific material are expected to create a text that is appealing and accessible to a wide audience, which involves making a selection of information to present, which serves his or her purpose - and thus the journalistic institutional goals (Goal 3 \& 4, Table 1) (Fahnestock, 1986: 281). Essentially, when creating a popular scientific text, an author manoeuvres between the institutional goals of adequacy and representation, while also attempting to maximally achieve the institutional goals of simplifying the material and indicating its societal importance and relevance, in order to ensure that is interesting to a broad public. As such, there exists a certain tension between the academic and journalistic institutional goals of popular scientific discourse, as it may not always be possible to maximally fulfil one without neglecting the other.

The adaptation of science communication aimed at an academic audience to communication that is intended for a lay audience represents a great shift in discursive context. As Jeanne Fahnestock remarks, "with a significant change in rhetorical situation comes a change in genre, and instead of simply reporting facts for a different audience [...] the work of science journalism requires the adjustment of new information to an audience's already held values and assumptions" (Fahnestock, 1986: 278-279). Thus, professional scientific material is not simply reported on in popular scientific discourse but is in fact recontextualized in the communicative activity type of popular science in order to ensure it fulfils both of its institutional goals. In that regard, a popular scientific text is a reinterpretation of scientific source material that must be argued for. However, in the popular scientific context of informing and persuading an audience of scientific fact — rather than offering scientific findings for critical evaluation - there is often no room for many qualifications towards what is being communicated. Although a smaller, academic audience may expect an author to provide a full justification for his or her statements and take a tentative approach to inferential claims, the wider audience has much less need for this type of information. Instead, a large and heterogeneous audience is mainly interested in the potential application of research or its appeal to a sense of wonder or progress (Fahnestock, 1986: 279). This particular audience demand is often met by the use of vivid imagery and presenting scientific claims with greater certainty than they may actually possess. Additionally, while the significance of what is being communicated in a professional scientific paper is largely understood and made clear contextually, this does not hold true for many popular scientific articles, which generally explicitly indicate the value of the scientific knowledge they report. Such practices tie back to the institutional goal of relating the societal importance of the scientific source material to the target audience, which is more likely to be adequately fulfilled with the use of these discursive strategies. 


\section{THE ARGUMENT FROM CORRELATION TO CAUSE}

\subsection{The statistical MEANing of a CORRElation}

Correlations fall within a broad class of statistical relationships that involve dependence and association between two or more variables. In its most common usage, a correlation between two variables refers to the extent to which these variables possess a linear relationship with each other. For example, the relation between the physical appearance of parents and the appearance of their children is a basic example of a correlation, since their relation is clearly dependent, but not completely linear. Correlations may also possess predictive capacities, as they can provide an indication of the likelihood a particular variable may occur. For instance, the weather conditions on a particular day are instrumental in assessing the required level of energy production a power plant should be prepared to meet. In that case, the correlation between extreme weather conditions and a corresponding increased demand for energy in order to heat or cool residences contains a causal relation. In other words, the weather conditions cause the increased need for energy, which means those weather conditions provide a solid indicator of energy demand. However, there are many other factors that may contribute to an increase in energy demand and extreme weather conditions do not always generate the same increase in energy demand, which may be the case during a holiday period. This means the overall relation of dependence between energy demand and weather conditions is still considered a correlation, while it has a causal relation embedded within its relation of dependence. In actuality, what is commonly subsumed under correlation is a broad range of different possible relations between variables.

When two variables or events - $A$ and $B$, for example - are correlated, there are a number of possible relationships that may exist between those variables, which include the following options (Govier, 2005: 288):

- $A$ causes $B$ (direct causation).

- $B$ causes $A$ (reverse causation).

- Both $A$ and $B$ are the consequences of a common cause $C$, however they have no causal relation towards each other. (Third-cause variable, "standard" correlation).

- There is in fact no actual connection between $A$ and $B$ (coincidence).

Because of the disparate nature of what a correlation may actually signify, ${ }^{1}$ a common statistical dictum is correlation does not imply causation.

${ }^{1}$ Theoretically, three more possible causal relations between the correlated variables could occur other than the ones mentioned by Trudy Govier (Govier, 2005: 288). $A$ and $B$ could also 
This means that on the basis of the fact that event $A$ and $B$ are correlated alone, one cannot draw a conclusion about the existence or the direction of a causal relationship between them. In order to establish whether any causal link between the observed variables exists, additional information is required. As such, the interpretation of a measured correlation as an indication of a certain causal connection is in fact a claim that requires argumentative support. If an arguer employs a correlation to validate the existence of a causal link, he or she can be reasonably expected to provide additional evidence that supports that particular interpretation over another. More specifically, an arguer claiming that a variable shares a causal relation with another may be required to adequately address the impossibility or unlikelihood of the other possible relations between those variables. In the case of an arguer claiming a direct causal relation - $A$ causes $B$ - on the basis of a correlation between them, he or she is using a specific type of argumentation that is also called an argument from correlation to cause (Walton, 1996: 71). However, given the fact that the correlation alone is not enough to substantiate a causal relation, the argument from correlation to cause should consist of multiple elements that must be adduced in defence of the causal claim in order to form a cogent inference. Section 3.2 to follow further elaborates on the expected argumentative pattern for arguments from correlation to cause.

\subsection{Argumentative pattern for the argument FROM CORRELATION TO CAUSE}

In previous research concerning the use of correlation in argumentation, several formulations of the argument from correlation to cause have been put forward. However, there are considerable differences between these formulations and their theoretical stance towards the reasoning behind the argument from correlation to cause. Douglas Walton (Walton, 1996: 71-73) formulates the argument from correlation to cause - which he baptized as such — in the following way:

Premise: $\quad$ There is a positive correlation between $A$ and $B$.

Conclusion: $\quad$ Therefore, $A$ causes $B$.

be the cause of one another, which is a cyclical causation. Also, $A$ could cause a third variable $C$, which in turn causes variable $B$, called an indirect causation. Although these relations are more complex, they include a causal relation from $A$ to $B$ and have been accommodated in that category. Lastly, $A$ and $B$ may both cause a third variable $C$, which is actively conditioned on, which is an example of Berkson's Paradox (Berkson, 1946). This category has been purposefully excluded, as it exceeds the theoretical scope of this article. 
Walton adds a number of critical questions that are aimed at identifying whether the relation invoked can be considered reasonable or not. He notes that "quite often, arguments fitting this argumentation scheme are presumptively correct. However, this type of argumentation tends to be very weak in many cases, because other factors are overlooked" (Walton, 1996: 71-72). But even if the argumentation employed in an argument from correlation to cause is weak, "the argument may still be a reasonable one, [...] because the critical questions could possibly be answered with further investigation of the case" (Walton, 1996: 72). Thus, it appears that in Walton's view, the argument from correlation to cause is not necessarily fallacious, but difficult to make inferentially valid.

In another treatment of argumentation from correlation to cause, Ralph H. Johnson and J. Anthony Blair (Johnson \& Blair, 1983: 121) subsume it under a broader category of arguments that employ a causal claim. As such, their treatment of the argument from correlation to cause departs from the category of post hoc and cum hoc fallacies, which are also called fallacies of questionable cause. Such fallacies have three identifying conditions: (1) there is a causal claim in the standpoint, (2) the protagonist fails to provide adequate evidence for the causal claim and (3) there are grounds to question the acceptability of the claim (Walton, 1996: 165). This interpretation of the argument from correlation to cause signals that Johnson and Blair do not consider such arguments inherently fallacious, seeing as a correlation — in combination with other arguments - could satisfy conditions (2) and (3).

Leo Groarke and Christopher Tindale (Groarke \& Tindale, 2004: 303) formulate an extensive argument scheme for the argument from correlation to cause, which is focused on representing the additional theoretical elements necessary to infer a causal relation on the basis of a correlation:

Premise 1: $\quad X$ is correlated with $Y$.

Premise 2: $\quad$ the correlation between $X$ and $Y$ is not due to chance.

Premise 3: $\quad$ the correlation between $X$ and $Y$ is not due to some mutual cause $Z$.

Premise 4: $\quad Y$ is not the cause of $X$.

Conclusion: $X$ causes $Y$.

On the basis of these earlier rendition of the argument and the necessary theoretical elements for this type of reasoning to be considered acceptable, my proposition for the expected argumentative pattern for the argument from correlation to cause is as follows:

We may assume that $A$ causes $B$. $A$ is correlated with $B$. 
1.1b A causal relation between $A$ and $B$ is plausible.

1.1c The correlation between $A$ and $B$ is not due to a common

1.1d $B$ does not cause $A$.

$\left(1.1 \mathrm{a}^{\prime}-1.1 \mathrm{~d}\right.$ ') $\quad$ If premises $1.1 \mathrm{a}$ through $1.1 \mathrm{~d}$ apply, we may assume that $A$ causes $B$.

$(1.1 \mathrm{a}-1.1 \mathrm{~d}$ ').1a If premises $1.1 \mathrm{a}$ through $1.1 \mathrm{~d}$ apply, $A$ is a necessary cause of $B$.

$(1.1 \mathrm{a}-1.1 \mathrm{~d}$ ').1b If premises $1.1 \mathrm{a}$ through $1.1 \mathrm{~d}$ apply, $A$ is a sufficient cause of $B$.

In this form, the argument from correlation to cause can be considered a subtype of causal argumentation (van Eemeren et al., 2002: 100-102). The standpoint under 1 that contains the claims to a causal relation between $A$ and $B$ is defended with a number of coordinative arguments, which only together constitute a complete substantiation of the claim. These arguments are provided in anticipation of critical questions that "relate to specific conditions under which a particular fact can be called the "cause of another fact" (Wagemans, 2016: 103). In support of the standpoint containing the causal claim, four coordinative arguments pertaining to the observed correlation between $A$ and $B$ can be adduced. Argument 1.1a contains the argument that there is a positive correlation between $A$ and $B$. Argument $1.1 \mathrm{~b}$ consists of any reason related to the plausibility of the suggested causal relation, such as circumstantial information regarding the evidence brought forward. In turn, argument $1.1 \mathrm{c}$ is adduced in order to exclude the possibility of a third variable causing both $A$ and $B$. Argument 1.1d eliminates the reverse and cyclical types of causal relations between the variables. These four coordinative arguments are aimed at the elimination of the existence of any other relation between the correlated variables $A$ and $B$, in order to support the standpoint containing the claim to a direct causation from $A$ to $B$. The implicit linking premise under (1.1a-1.1d') reflects the arguer's assumption that the arguments mounted in defence of the direct causal relation provide an adequate substantiation of the causal claim. In theory, this argument scheme for the argument from correlation to cause represents all the necessary elements for a valid and acceptable inference, in which arguments $1.1 \mathrm{a}$ through $1.1 \mathrm{~d}$ each provide a piece of the puzzle. Finally, the implicit linking premise may be further supported by two coordinative argument relating to the sufficiency and necessity of the causal relation between $A$ and $B$. Argument (1.1a'-1.1d').1a states that the causal relation from $A$ to $B$ is necessary, which means that "without the occurrence of the cause, the effect does not occur either" (Wagemans, 2016: 103). Argument (1.1a'-1.1d').1b states that $A$ is a sufficient cause of $B$, which means that no other factors such as a third variable - are needed for the effect to occur. Section 3.3 to 
follow will stipulate the criteria for the reasonableness of arguments from correlation to cause.

\subsection{Formulating CRITICAL QUESTIONS FOR THE ARgUMENT FROM CORRELATION TO CAUSE}

The criteria for reasonable arguments from correlation to cause can be formulated as a number of critical questions that examine the argument's acceptability. Since the argument from correlation to cause is a subtype of causal argumentation, the central critical question that must be answered is "does $A$ really lead to $B$ "? In the specific case of reasoning from a correlation to a causal relation, reaching an answer to this question can be achieved by posing a number of sub-questions. These critical questions can be subdivided into two main categories: critical questions aimed at critically examining the propositional content of the argument and critical questions aimed at validating the inferential structure of the argument. I have formulated five critical questions that pertain to the propositional content of the argument as follows:

Critical Question 1: Is there a positive or negative correlation between $A$ and $B$ ?

Critical Question 2: Is the correlation between $A$ and $B$ significant?

Critical Question 3: Is the correlation between $A$ and $B$ not due to mere chance?

Critical Question 4: Can we rule out that there is a third variable responsible for theobserved correlation between $A$ and $B$ ?

Critical Question 5: Can we rule out that $B$ causes $A$ ?

The first three critical question are aimed at establishing whether there is indeed a positive correlation between the variables, and if that correlation is significant and not due to coincidence. Question 1 can be answered by producing the data the claim to a correlation between $A$ and $B$ is based on, while Question 2 and 3 require a substantiation of the interpretation of that data as a meaningful and significant correlation. For instance, when two traits of human behaviour are observed to be correlated, Question 2 may be answered by indicating that the observed correlation between those variables is strong enough to be significantly different from the norm and thus may be considered representative for the entire population. Question 3 is generally addressed by the use of a repeated measurement design, which allows for the exclusion of a coincidental correlation on the basis of observing the correlation multiple times.

Question 4 and 5 aim to further complete the necessary grounds for an inference from correlation to cause. The exclusion of a third variable that may be 
responsible for the measured correlation is one of the most difficult conditions to meet, since many causal relations are open to alternative explanations. This question may be addressed by attempting to eliminate the most plausible other options for the observed correlation between $A$ and $B$. In turn, Question 5 is not always a necessary critical question, since the exclusion of a causal link from $B$ to $A$ may already be obvious on the basis of our background knowledge about the world. For example, in a correlation between the speed at which windmills spin and the strength of the wind, a reverse causation can be excluded because of the understanding that windmills are not the cause of the wind. However, in the case of a correlation between more complex variables - such as unemployment and slow economic growth, for example - a reverse causation is equally plausible, as either could potentially cause the other. In more complex cases, Question 5 must be answered with a further substantiation for the direction of the causal relation.

I have also formulated three possible critical questions that are aimed at examining the legitimacy of the inference made in an argument from correlation to cause:

Critical Question 6: Do premises 1.1a through 1.1d provide an adequate substantiation of a causal relation from $A$ to $B$ ?

Critical Question 7: Do any exceptions to the causal relation apply, and if so, can they be clearly delineated as exceptions?

Critical Question 8: When a premise in the argument is missing, does this prevent a valid inference to a causal relation?

Question 6 is centred on the evaluation of the implicit linking premise of the argument from correlation to cause, which is the assumption that the coordinative arguments $1.1 \mathrm{a}$ through $1.1 \mathrm{~d}$ in fact constitute an adequate defence of a causal claim. In some cases, it may be necessary to adduce additional arguments in order to fully substantiate the causal claim in the standpoint. For example, in the case of an indirect causation between $A$ and $B-$ this occurs when $A$ causes $C$, which in turn causes $B$ - the argument's protagonist may be expected to further elaborate on the intervening variable (Walton, 1996: 72). Question 7 aims to uproot any exceptions to the causal relation that is invoked in the standpoint and requires an explanation for their exceptional status. In response, the argument's protagonist could answer by indicating the ranges in which the causal relation between variable $A$ and $B$ does hold. Lastly, Question 8 is an especially important question to consider in the case of partially incomplete arguments from correlation to cause. As mentioned with regard to critical Question 5, sometimes not all of the arguments listed in the argument scheme for the argument from correlation to cause are necessary for the argument to be acceptable. Also, as Walton points out, an argument 
from correlation to cause overlooking or failing to account for one or more of these critical questions does not necessarily entail that it is fallacious (Walton, 1996: 72). As such, determining whether an argument from correlation to cause is reasonable must proceed on the basis of both critical questions relating to the propositional content of the argument, as well as the critical questions that examine its inferential acceptability.

\section{CORRELATION TO CAUSE ARGUMENTATION IN POPULAR SCIENCE}

Using the argumentative pattern outlined in Section 3 as a blueprint, it is now possible to analyse an argument from correlation to cause within the context of science communication. The expert-to-expert scientific and peer-reviewed journal for Behavioural Brain Research has recently published a study focussed on the relation between Facebook usage and brain structure by Christian Montag et al. (Montag et al., 2017: 221-228) titled Facebook usage on smartphones and grey matter volume of the nucleus accumbens. The study used a remote smartphone activity tracker to record the actual Facebook activity of 62 participants and correlated this data with the volume of the nucleus accumbens in the brain. The nucleus accumbens is a region in the brain also known as its "reward centre", and likely plays an important role in a wide range of positive experiences. The resulting data of the study indicate that "in particular higher daily frequency of checking Facebook on the smartphone was robustly linked ${ }^{2}$ with smaller grey matter volumes of the nucleus accumbens" (Montag et al., 2017: 221). In this case, the correlation was negative: "participants who opened the Facebook application more frequently and participants who stayed on Facebook longer had smaller nuclei" (Montag et al., 2017: 224). However, the authors stipulate that "given the cross-sectional design of the present study, it remains to be elucidated whether lower volumes of the accumbens constitute a factor for increased social media use or whether it results as a consequence of higher usage" (Montag et al., 2017: 226). In other words, the study has found the variables to be strongly correlated but has not been able to establish the existence or direction of a possible causal link between the measured variables.

2 "Robustly linked" means as much as "strongly correlated" in this context. In relation to this discursive phenomenon, Govier points out that "the word linked [...] enables writers to skirt over the distinction between correlational evidence and causal claims conclusions, because it has several meanings that are rarely distinguished from each other. To say that $\mathrm{Q}$ is linked to $\mathrm{H}$ suggests, but does not assert, a causal connection between $\mathrm{Q}$ and $\mathrm{H}$. Because the connection is only suggested, the demands of a rigorous causal argument are avoided. But because the connection is suggested, there is a clear implication that something more than a correlation has been established" (Govier, 2005: 290, italics in original). 
I have formulated a reconstruction of this professional scientific publication's most relevant argumentation below:

1 The nucleus accumbens could be involved in online social media usage.

1.1a Higher daily frequency of checking Facebook is robustly linked with smaller grey matter volumes of the nucleus accumbens.

1.1a.1 Left and right nucleus accumbens volume is negatively correlated with Facebook usage.

1.1b The results from other neuroscientific studies suggest that the rewarding aspects of Facebook usage could result in over-usage of this platform.

1.1c Data show that the frequency of daily Facebook checking is also associated with self-reported tendencies towards online social network addiction.

The claim Montag et al. make on the basis of their observed correlation is careful, and states no more than a possible involvement of the two variables investigated (Montag et al., 2017). The claim is supported by the observed negative correlation between the variables and other coordinative argumentation that suggests other evidence for the plausibility of the connection.

In the "Health" section of Rush Hour Daily news, these professional scientific findings are popularized in an article that claims, "Facebook addiction causes decrease in brain's grey matter" (Ulaky, 2017). The article goes on to report the measured negative correlation between Facebook usage and nucleus accumbens volume, as well as another study concerning the use of Facebook and markers of general well-being. Furthermore, the popular scientific article contends that researchers Montag et al. used magnetic resonance imaging (MRI) scans, which "ultimately showed the negative reaction too much social activity might have" (Ulaky, 2017). My reconstruction of the correlation to cause argumentation employed in the popular scientific article is as follows:

1 Increased Facebook usage leads to a decrease in grey matter in the brain.

1.1 A study published in the journal Behavioural Brain Research has discovered the negative side effects and psychological repercussions of Facebook "addiction".

1.1.1a People who frequently check the application on their smartphones are likely to have less grey matter the "reward centres" of their brains.

1.1.1a.1 Higher daily frequency of checking Facebook is robustly linked with smaller grey matter volumes of the nucleus accumbens. 
1.1.1b MRI-scans of the test subjects showed the negative reaction too much social activity might have.

From an evaluative point of view, the reconstructed argument from correlation to cause in Rush Hour Daily's article can be considered fallacious on two accounts:

Firstly, the representation of the scientific source material the article is based on is inadequate and unreasonable towards the original authors. In argument 1.1, the author of the popular scientific article states that researchers Montag et al. have "discovered the negative side effects and psychological repercussions" to the use of Facebook, which signals a relation of cause and effect between the variables. However, Montag et al. explicitly indicate they have not yet discovered the substantive nature of the correlation they have observed (Montag et al., 2017: 226). In that regard, the popularized article commits a strawman fallacy, by implying the study by Montag et al. has claimed a causal relation between the correlated variables. In doing so, the popularized article violates the third pragma-dialectical rule for critical discussion, which states that an arguer may not distort or misrepresent the standpoint of another party.

Secondly, the inference from correlation to cause made in the popular scientific article is unacceptable, since it is improperly substantiated with evidence other than a correlative argument. Argument 1.1.1b is used in the article to support the interpretation of the original study as a relation of cause and effect. In reality, the MRI-scans of the test subjects' nucleus accumbens indicated the correlation of its volume and Facebook usage, but no causal relation. As such, the correlative argument 1.1.1a and the incorrect interpretation of the data in argument 1.1.1b entail the fallaciousness of this argument from correlation to cause. The author commits the fallacy of cum hoc ergo propter hoc, by making the assumption that the observed variables co-occurring allows for a causal conclusion. The reasoning in the popularized article does not effectively rule out alternative explanation before making a causal claim and does not acknowledge that the remaining premises alone do not adequately substantiate the claim.

This example demonstrates the tension between the academic and journalistic institutional goals a popular scientific article aims to fulfil. By presenting the observed connection between Facebook usage and grey matter volumes in the brain as a causal relation, this article has very likely achieved both journalistic institutional goals of simplification and indicating significance. This is further abetted by the nature of the topic, which is of wide application and interest, which now also generates a cause for concern. Although popular science writers "must usually be explicit about the value of scientific discoveries, [as] they cannot rely on the audience to recognize the significance of information" (Fahnestock, 1986: 279), in the example this has been achieved at the expense 
of the institutional goal of adequacy and representation. In sum, the author of this particular popular scientific article appears to have placed too great an emphasis on fulfilling the institutional goals situated within the journalistic domain of communication, thereby overstepping the reasonable bounds for critical discussion.

\section{CONCLUSION}

With this paper, I have endeavoured to develop an argumentation theoretical basis for the analysis and evaluation of arguments from correlation to cause within the context of science communication. In Section 2, I have briefly discussed the communicative characteristics of popular scientific texts. Section 3 focussed on formulating the expected argumentative pattern for arguments from correlation to cause and a number of critical questions for assessing their acceptability, and Section 4 discussed a natural example of argumentation from correlation to cause within the context of science communication. The argumentative use of correlation and cause plays an important role in the process of obtaining valid and substantiated scientific explanations for the relation between observed phenomena. While correlations may provide an important hint to the existence of a causal relation, they do not license a causal claim on their own. An acceptable argument from correlation to cause must contain a number of additional elements that support the inferential step from a correlation to a relation of cause and effect.

The exclusion of the possible effects of external variables in an argument from correlation to cause is the subject of debate in numerous scientific fields. In many behavioural and medical research projects, the total exclusion of tertiary factors that may be of influence on an observed correlation can be very difficult - or even impossible. For those cases, it may not be achievable to assemble a complete argument from correlation to cause, but that does not necessarily make the scientific findings any less valuable. The precise conditions under which a causal connection can be considered adequately or sufficiently established affect not only the academic world, but also the realms of government and politics. For example, if a particular product is found to be correlated with a risk to health, the conditions that stipulate when a legitimate inference from a correlation to cause may be made are instrumental in determining when a government is allowed to intervene. In view of this, it would prove conducive to precisely identify the argumentative conditions under which external variables are generally considered acceptably excluded in establishing causality.

There also remain a number of interesting directions for future research on this topic, one of which is providing non-expert audiences with a blueprint for the critical assessment of claims on the basis of correlations in popularized 
scientific texts. Since the intended audience of popular scientific discourse is highly diffuse, its contents and aims may vary greatly per category of popularization. For example, intra-scientific popular discourse is intended for members of the academic world active in other fields of research and results in entirely different popular scientific material than popularized articles in a newspaper or a magazine dedicated to popular science for a general audience. It would be fruitful to characterize each of these subtypes of scientific popularization as a communicative activity type in order to chart how the variations in intended audience influence their respective institutional goals and conventions. The set of critical questions set out in this paper can contribute to reducing the risk of misinformation by uncareful or premature claims in popularized scientific material ${ }^{3}$.

\section{BIBLIOGRAPHY}

Altman, N. \& Krzywinski, M. (2015). Points of significance: Association, correlation and causation. Nature Methods, 12, 899-900.

Berkson, J. (1946). Limitations of the application of fourfold table analysis to hospital data. Biometrics Bulletin, 2, 47-53.

van Eemeren, F.H. (2010). Strategic manoeuvring in argumentative discourse. Extending the pragma-dialectical theory of argumentation. Amsterdam-Philadelphia: John Benjamins.

van Eemeren, F.H. \& Grootendorst, R. (1984). Speech acts in argumentative discussions. A theoretical model for the analysis of discussions directed towards solving conflicts of opinion. Dordrecht-Berlin: Foris Publications / Mouton de Gruyter.

van Eemeren, F.H., Grootendorst, R., \& Snoeck Henkemans, F. (2002). Argumentation: Analysis, evaluation, presentation. Mahwah-London: Lawrence Erlbaum Associates.

van Eemeren, F.H. \& Grootendorst, R. (2004). A systematic theory of argumentation: The pragma-dialectical approach. Cambridge: Cambridge University Press.

van Eemeren, F.H., Garssen, B., Krabbe, E., Snoeck Henkemans, F., Verheij, B., \& Wagemans J. (2014). Handbook of argumentation theory. Dordrecht-Heidelberg-New York-London: Springer.

Fahnestock, J. (1986). Accommodating science: The rhetorical life of scientific facts. Written Communication, 3, 275-295.

Govier, T. (2005). A practical study of argument. (6th ed.). Belmont: Wadsworth.

Groarke, L.A. \& Tindale, C.W. (2004). Good reasoning matters!. (3rd ed.). Oxford: Oxford University Press.

Jamieson, K. \& Kahan, D., \& Scheufele, D. (Eds.). (2017). The Oxford bandbook of the science of science communication. Oxford: Oxford University Press.

Johnson, R.H. \& Blair, J.A. (1983). Logical self-defense. (2nd ed.). Toronto: McGraw-Hill Ryerson.

\footnotetext{
${ }^{3}$ I would like to thank Jean H.M. Wagemans for his many insightful comments and suggestions on earlier drafts of this work, and my thanks go out to Katarzyna Budzyńska and Marcin Koszowy for their help and advice in preparing the paper for publication. I am also grateful to two anonymous reviewers for their close reading of the manuscript and their helpful comments to improve it.
} 
Shinn, T. \& Whitley, R.P. (Eds.). (1985). Expository science: Forms and functions of popularisation. Dordrecht: Reidel.

Wagemans, J.H.M. (2016). Argumentative patterns for justifying scientific explanations. Argumentation, 30, 97-108.

Walton, D.N. (1996). Argumentation schemes for presumptive reasoning. Mahwah: Erlbaum.

Walton, D.N. (2015). Argument evaluation and evidence. Dordrecht-Heidelberg-New YorkLondon: Springer.

Walton, D.N., Reed C., \& Macagno, F. (2008). Argumentation schemes. New York: Cambridge University Press.

Montag, C., Markowetz, A., Blaszkiewicz, K., Andone, I., Lachmann, B., Sariyska, R., Trendafilov, B., Eibes, M., Kolb, J., \& Reuter, M. (2017). Facebook usage on smartphones and gray matter volume of the nucleus accumbens. Behavioural Brain Research, 329, 221-228.

Ulaky, S. (2017). Facebook addiction causes decrease in brain's gray matter, new study reveals. Rush Hour Daily News, 29.05. 
